\title{
Periodic Pion-Pion Scattering at the Physical Point: Update
}

\section{Daniel Hoying ${ }^{* \dagger}$}

Brookhaven National Laboratory, 20 Pennsylvania St., Upton, NY, 11973;

University of Connecticut, 2152 Hillside Road, unit 3046, Storrs, CT, 06269

E-mail: daniel.hoying@uconn.edu

\begin{abstract}
We present updated results on the scattering of pseudoscalar, vector, and scalar mesons on a physical pion mass, $2+1$ flavor mobius-DWF, ensemble with periodic boundary conditions (PBCs) generated by the RBC and UKQCD collaborations. Using all-to-all propagators, we produce thousands of correlator momentum combinations. Energy spectra and phase shifts, including excited states, are then extracted via the solutions of a generalized eigenvalue problem. Included in this paper will also be an overview of improved analysis techniques and a second lattice spacing. These studies are intended to serve as groundwork for a full PBC calculation of direct CP violation in kaon decays.
\end{abstract}

The 37th Annual International Symposium on Lattice Field Theory - LATTICE2019

16-22 June, 2019

Wuhan, China

\footnotetext{
* Speaker.

${ }^{\dagger}$ This work was done in collaboration with members of the RBC and UKQCD Collaborations.
} 


\section{Introduction}

Our calculation is one of three RBC/UKQCD collaboration calculations of pion scattering at the physical point, removing an important systematic from previous (e.g. [1][2]) calculations. We would also like to use our calculation (which uses periodic boundary conditions) to check the $K \rightarrow \pi \pi$ and $\pi \pi \rightarrow \pi \pi$ G-parity calculations used to determine from first principles the important Standard Model (SM) ratio $\varepsilon^{\prime} / \varepsilon$. In particular, our calculation aims to extract infinite volume scattering phase shifts via the Lüscher formula [3]. Physical kinematics is extracted from an excited state in each case.

\section{Correlator Production}

We calculate many correlation functions at lattice spacing $1.015,1.3784 \mathrm{GeV}\left(24^{3} \mathrm{DSDR}\right.$ and $32^{3}$ ensembles respectively at identical physical volumes but both with $L_{t}=64$ ) on Intel Knight's Landing (KNL) clusters using all-to-all propagators [4] and (Zmöbius, Möbius) Domain Wall fermions. In order to save on computational resources, we use all-mode-averaging (AMA) to compute on a limited number of gauge configurations exactly and the rest in an inexact way that introduces some small systematic errors which are corrected using the exact measurements). We time-split our operators so that one pion is forced to propagate for 3-4 time slices before we create/destroy the other pion since this is smaller overlap with the vacuum state for isospin $I=0$. We recover the full point-to-point propagator in the large noise sample limit. We then project our $\pi \pi$ operators onto definite isospin ( $I=0,1,2$; isospin is a good symmetry in our simulation). We calculate many momentum combinations up to three units of lattice momenta for an individual meson (and up to three units of center of mass momentum), so that we can project onto definite irreducible representations (irreps) of the group of lattice rotations. We denote center of mass frame, one, two, and three units of center of mass momentum by (respectively) $p 0, p 1, p 11, p 111$. This symmetry is reduced from the full rotational symmetry group because of lattice discretization. As a result, we project onto a tower of spin states, but we assume (and have seen some evidence to support) all higher spins are suppressed relative to $J=0$ for our $I=0,2$ irrep and $J=1$ for our $I=1$ irrep.

We calculate beyond the $4 \pi$ threshold, but again we assume (and have seen some empirical evidence to support the idea) that these contributions are suppressed relative to $\pi \pi$ contributions. We have this caveat because the formalism to extract continuum phase shifts from inelastic processes (such as $\pi \pi \rightarrow 4 \pi$ ) does not exist.

\section{GEVP, Operator Basis}

Our isospin/irrep projected operators have Dirac-gamma structure $\bar{\psi} \gamma_{5} \psi$ ( $\pi$ operator), $\bar{\psi} \gamma_{\mu} \psi$ ( $\rho$ operator, $I=1$ ), and $\bar{\psi} \psi(\sigma$ operator, $I=0)$. Our $\rho$ (vector) operator has angular momentum, so we also need to properly project the $\gamma_{\mu}$ polarizations into the proper irrep (row).

For $I=0$ we also need to perform vacuum subtraction for disconnected diagrams by subtracting from our (jackknifed) gauge configuration sample the averaged (over gauge configurations, but not time) bubble. We then multiply source and sink subtracted bubbles to compose the disconnected diagrams. 
We diagonalize our Hamiltonian (up to excited state effects) via the solution of a generalized eigenvalue problem (GEVP): $C(t) v\left(t, t_{0}\right)=\lambda\left(t, t_{0}\right) C\left(t_{0}\right) v\left(t, t_{0}\right)$ where we fix $t-t_{0}=$ const. where $C(t)$ is a matrix of lattice correlation functions composed of operators projected onto a particular irrep/isospin and at source, sink separation $t$. We obtain an asymptotic error $O\left(e^{-\left(E_{N+1}-E_{n}\right) t}\right)$ for an $N$ dimensional GEVP for energy $E_{n}$ [5] (see [6] for further matrix element developments we plan to use for the full periodic $K \rightarrow \pi \pi$ calculation).

\section{Removal of Systematic Error Via $\pi \rightarrow \pi$ Correlation Functions}

\subsection{Around the World Contamination - Vacuum Saturation Subtraction}

In any two particle lattice simulation, the finite size of the box necessarily generates aroundthe-world (ATW) contamination caused by one particle propagating forward in time and the other backward. Since the particles do not share too many time slices, one can expect that most of this effect can be approximated by the non-interacting case. If each single particle correlation function has (Euclidean) time dependence $C_{n}(t)=e^{-E_{n} t}+e^{-E_{n}(L t-t)}$ and $C_{m}(t)=e^{-E_{m} t}+e^{-E_{m}(L t-t)}$ then the non-interacting ATW piece is $e^{-E_{n} t} e^{-E_{m}(L t-t)}+e^{-E_{n}(L t-t)} e^{-E_{m} t}$. We can see that this term is constant with respect to time if $E_{m}=E_{n}$. Moreover, if scattering identical particles in the center of mass frame, all ATW terms including the interacting terms are necessarily constant by exchange symmetry. If this is the case, we can perform a GEVP matrix subtraction to entirely remove this effect. We define $D(t) \equiv C(t)-C\left(t-\delta_{t}\right)$ and solve a modified GEVP $D(t) v\left(t, t_{0}\right)=\lambda\left(t, t_{0}\right) D\left(t_{0}\right) v\left(t, t_{0}\right)$ (at the cost of losing $\delta_{t}$ time slices from our fitting range).

If instead $E_{m} \neq E_{n}$, (e.g. in the case of moving frames) then the ATW terms become time dependent. In the case of the $N$-dimensional GEVP, we get $2 N$ non-interacting terms (in addition to interacting time dependent terms). The non-interacting terms can be removed via iterated matrix subtraction, but this is not an economical use of time slices. Instead, we perform Vacuum Saturation Subtraction which removes the non-interacting terms entirely by (roughly speaking) crossmultiplying the exponential pieces of the cosh of the averaged single particle correlators. Details of this procedure can be found in [7]. We should mention that this apparently results in improved agreement with $I=2$ Roy equation results, but does not substantially correct the problem for all $I=0$ moving frames (particularly in the $32^{3}$ ensemble which has a physically smaller time extent).

\subsection{Discretization Error - Pion Ratio Method}

We note that squaring the averaged single-pion correlation function (being careful about isospin pieces and time-splitting) gives us the energy of the non-interacting $\pi \pi$ system (defined to be

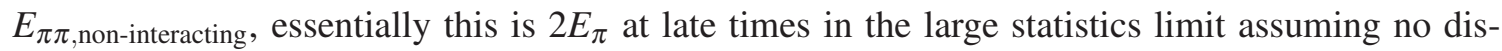
cretization error). We can also obtain this energy from the pion mass via the continuum dispersion relation $2 E_{\pi}=2 \sqrt{m_{\pi}^{2}+p^{2}}$. We naturally expect that the latter formula has less discretization error, so if we add $2 \sqrt{m_{\pi}^{2}+p^{2}}-E_{\pi \pi \text {,non-interacting }}$ to our (interacting) $E_{\pi \pi}$, we obtain less discretization error. As an added bonus, we obtain up to a factor of 10 noise reduction in all isospin channels due

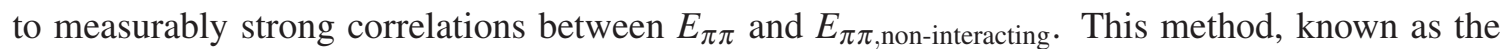
pion ratio method (for historical reasons), is due primarily to X. Feng (initial idea) and C. Lehner (current form) with development and testing from D. Murphy and the author. 


\section{Fitting Procedure (Outline)}

We perform a simultaneous fit to the effective masses of the GEVP, looping over fit ranges, randomly sampling 16 different fit ranges (which empirically gives stable weighted averages) only if the number of possibilities is combinatorially too large. We display in sections 6 and 7 the p-value weighted averages of these results.

The $I=2$ plot displays a shaded tolerance band representing a $1 \sigma$ uncertainty on the fit parameter. Energies displayed are in lattice units. Lines from the dispersion relation $\sqrt{m^{2}+p^{2}}$ are also plotted corresponding to the operator momenta used. The number of guage configurations is displayed in the title as "total, exact", e.g. 185,7 represent 185 total gauge configurations, 7 of which are also calculated exactly in order to perform the sample AMA procedure.

Straight lines with arrows joining the $24^{3}$ and $32^{3}$ ensemble results. The arrows point in the direction of the $32^{3}$. Because of slightly heavier pion mass in the $32^{3}$, the arrows always flow from left to right. Given that both points should be the same state (at two different lattice spacing), we should expect that the arrows should roughly point in the direction of the continuum limit.

We fit to different subsets of the data (referred to as different fit ranges). The number of such subsets is often combinatorially explosive, so we make data quality cuts and randomly sample (if the remaining amount is still too large). These cuts include demanding that three points are fit per energy level, and the points form an arithmetic sequence in (Euclidean) time (only regular sparsenings are allowed). We then perform a fully correlated (with respect to operators and time slices) fit, minimizing $\chi^{2}$. If the errors are gaussian, we can find the p-value from Hotelling's $t^{2}$ distribution (comparisons to the exact p-value give good empirical agreement). We do so, then average the different fit range results weighted by this $\mathrm{p}$-value.

For $I=2$, we fit to a constant, while for $I=0$ we fit to $E_{n}+a_{n} * \exp \left(-\left(E_{N+1}-E_{n}\right) t\right)$ with $2 N+1$ free parameters (for $N$ operators: $a_{n}, E_{n}, E_{N+1}$ ). 


\section{6. $I=2$ (Preliminary)}

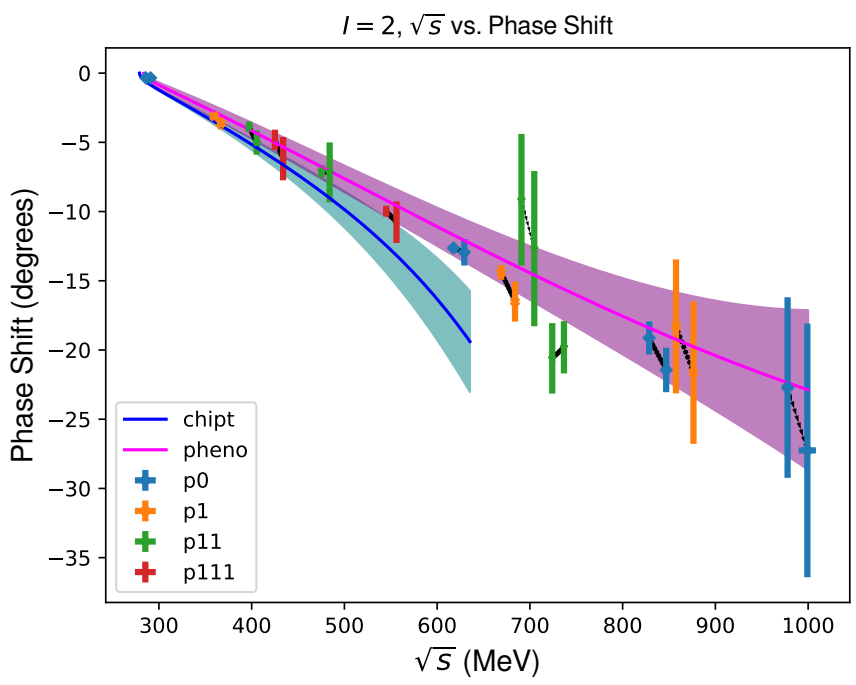

Figure 1: $I=2$ scattering phase shifts vs. Mandelstam $\sqrt{(s)}$.

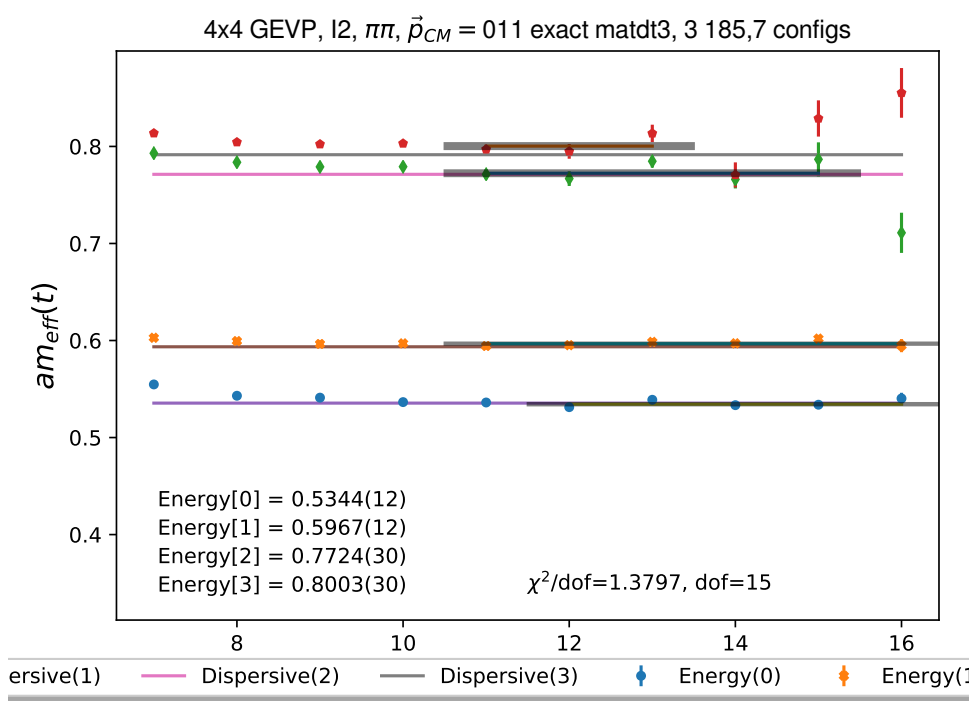

Figure 2: Representative effective mass plot for $I=2$ : two units center of mass momentum, four operators consisting of pions (hydrogen-like spatial wave function of $\bar{\psi} \gamma_{5} \psi$ ) with all possible lattice momentum combinations up to three units of individual momenta. $\delta_{t}=3$ for two matrix subtractions. 185 sloppy configurations are used with 7 of these are also calculated exactly. '2xjk' means we fit (to a constant) under a jackknife. 


\section{7. $I=0$ (Preliminary)}

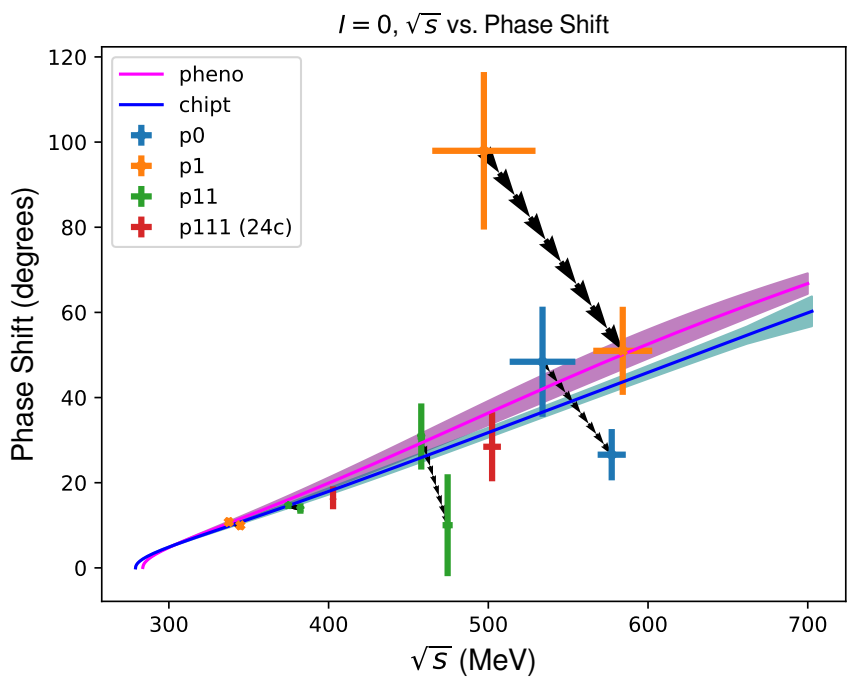

Figure 3: $I=0$ scattering phase shifts vs. Mandelstam $\sqrt{(s)}$.

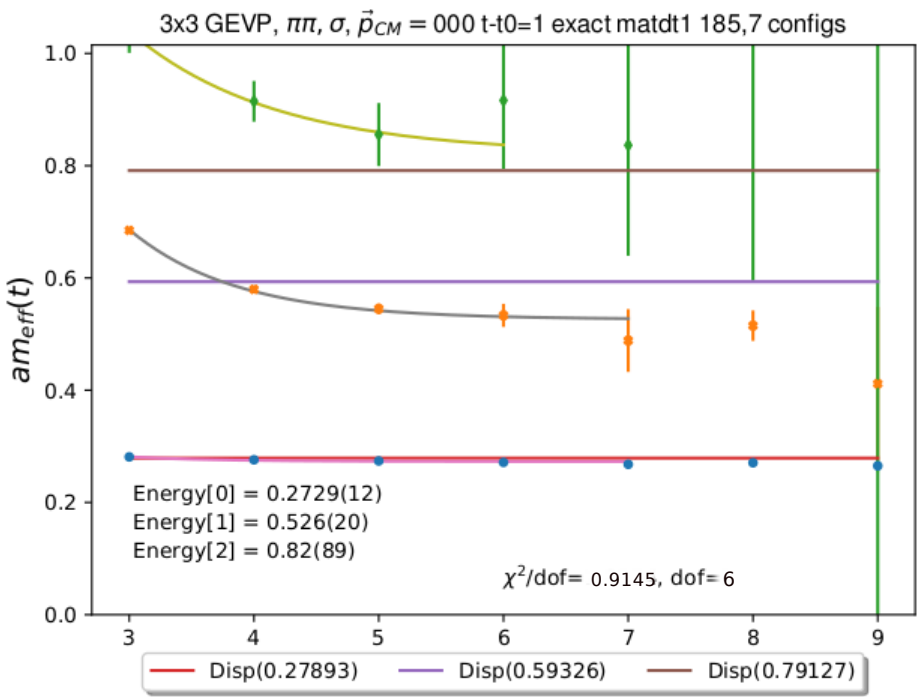

Figure 4: Representative effective mass plot for $I=0$ : center of mass frame, one stationary (hydrogen-like spatial wave-function with gamma structure $\bar{\psi} \psi$ ) operator, and two two-pion (hydrogen-like spatial wave function with gamma structure $\bar{\psi} \gamma_{5} \psi$ ) operators (one operator at rest, one with one unit of back-to-back lattice momentum). $\delta_{t}=1$ for two matrix subtractions; $t-t 0=1.185$ sloppy configurations are used with 7 of these are also calculated exactly. '2xjk' means we fit (to a const+exp) under a jackknife.

\section{Analysis}

The above results are preliminary, but roughly represent what will likely be final values we expect to publish soon. The summary plots (sections 6 and 7) display the p-value weighted averages 
(see section 5). For $I=2$, good plateaus are observed, so GEVP energies are fit to a constant, whereas for $I=0$ and 1 the energies are fit to a constant plus exponential term to deal with the obvious excited state contamination; (see section 3).

Compared to the Roy equation analysis (from M. Bruno; priv. comm.) we see good agreement for $I=2$, reasonable agreement for $I=0$, and only partial agreement for $I=1$. In the latter case our results lie significantly above the Roy analysis above the resonance region. Chiral perturbation theory results from D. Murphy (priv. comm.) are also displayed and agree well with the lattice results (and the Roy equation analysis) at low energies.

Two other physical point $\pi \pi$ scattering calculations are also nearing completion (the G-parity and Distillation calculations mentioned above; see talks/proceedings of this conference of T. Wang, A. Meyer, and C. Kelly).

\section{References}

[1] J. J. Dudek, R. G. Edwards and C. E. Thomas,

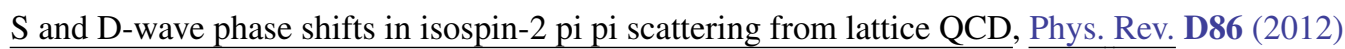
$034031[1203.6041]$.

[2] CP-PACS collaboration, T. Yamazaki et al., $\underline{I=} 2$ pi pi scattering phase shift with two flavors of $\mathrm{O}(\mathrm{a})$ improved dynamical quarks, Phys. Rev. D70 (2004) 074513 [hep-lat/0402025].

[3] M. Luscher, Two particle states on a torus and their relation to the scattering matrix, Nucl. Phys. B354 (1991) 531.

[4] J. Foley, K. Jimmy Juge, A. O'Cais, M. Peardon, S. M. Ryan and J.-I. Skullerud, Practical all-to-all propagators for lattice QCD, Comput. Phys. Commun. 172 (2005) 145 [hep-lat/0505023].

[5] B. Blossier, M. Della Morte, G. von Hippel, T. Mendes and R. Sommer, On the generalized eigenvalue method for energies and matrix elements in lattice field theory, JHEP 04 (2009) 094 [0902.1265].

[6] J. Bulava, M. Donnellan and R. Sommer, On the computation of hadron-to-hadron transition matrix elements in lattice QCD, JHEP 01 (2012) $140[1108.3774]$.

[7] D. Hoying, Improved Pion Correlation Functions Under Lattice Action, Ph.D. thesis, University of Connecticut, 2019. https://opencommons.uconn.edu/dissertations/2146/. 